\title{
Mean difference of Electrolyte Level measured in Arterial Blood gases and measured in Laboratory in Children presenting in Pediatric Intensive Care Unit
}

Hafiz Muhammad Shahid Shafique, ${ }^{1}$ Muhammad Umar Shafiq, ${ }^{1}$ Shahjhan Fazlani, ${ }^{1}$ Shoaib Ali, ${ }^{1}$ Aaqib Javed, ${ }^{1}$ Afifa Munir

Ahmad $^{1}$

\begin{abstract}
Background: Electrolytes are measured in arterial and venous blood by arterial blood gas analyzer and the autoanalyzers respectively.

Objective: To determine the mean difference in electrolyte level in arterial blood gases (ABGs) level versus laboratory serum electrolyte level in the children admitted in the pediatric intensive care unit.

Methodology: This cross-sectional study was conducted at department of Pediatrics, the Children's Hospital and Institute of Child Health, Lahore from 1st December 2015 to 31st May 2016. Total of 125 children fulfilling inclusion criteria were enrolled in the study from pediatric intensive care unit. The ABGs (whole blood) electrolytes were obtained immediately after collection, using ABGs analyzer. Serum electrolytes were analyzed in the central laboratory of the institution. Reports were assessed and levels of sodium and potassium was noted from reports of ABGs and laboratory.

Results: Mean $\mathrm{NA}^{+}$value on $\mathrm{ABG}$ and from laboratory was 134.66 and $132.26(\mathrm{p}=0.01)$. Mean $\mathrm{K}^{+}$value from on $\mathrm{ABGs}$ and from laboratory was 4.51 and 4.28. ( $\mathrm{p}=0.071$ ). In age group 1-5 and 6-10 years, $\mathrm{K}^{+}$level was high in $\mathrm{ABGs}$ value ( $\mathrm{p}$-value $=0.065 \& \mathrm{p}$-value $=0.073$ ). However, in age group 11-15 years $\mathrm{K}^{+}$level was significantly higher in ABGs value as that of laboratory value ( $\mathrm{p}$-value $=0.014$ ). The same trend was observed in male and female children that mean $\mathrm{NA}^{+}$and $\mathrm{K}^{+}$value with ABGs was significantly higher as compared to that of laboratory value.

Conclusion: $\mathrm{NA}^{+}$and $\mathrm{K}^{+}$in arterial blood gases level were different from laboratory serum electrolyte level in children admitted to pediatric intensive care unit. Clinician should be aware of differences so that potential misdiagnosis does not occur and unnecessary treatment or investigation can't be performed.
\end{abstract}

Keywords: Electrolyte level, Arterial blood, Laboratory, Serum electrolyte, Intensive care unit

Article Citation: Shafique HMS, Shafiq MU, Fazlani S, Ali S, Javed A, Ahmad A.M. Mean difference of Electrolyte Level measured in Arterial Blood gases and measured in Laboratory in Children presenting in Pediatric Intensive Care Unit. JSZMC 2021;12(2):03-07.

DOI: https://doi.org/10.47883/jszmc.v12i02

This Open Access Article in Journal of Sheikh Zayed Medical College is licensed under a Creative Commons Attribution- 4.0 International License(CC BY 4.0).

\section{Introduction}

In the intensive care unit (ICU), according to the patient's condition, it is often necessary to perform investigations quickly and do management of the patients according to investigations._Electrolytes are measured in arterial and venous blood by arterial blood gas analyzer and the auto-analyzers respectively. In the intensive care unit, according to the patient's condition often it is necessary to monitor biochemically with indicative laboratory investigation and prompt management. The pointof-care tests (POC), also called patient's bedside tests,. Conventionally, these tests were performed in the main laboratory of the hospital. ${ }^{1}$ With the advancement in technology, The point-of-care tests (POC ), vibrant work in advancement; the analyzers are convenient, smaller, quicker, and more friendly user and have been established to obtain precision with ever-smaller blood samples, aiding in blood conservation. ${ }^{1-3}$

Point-of-care ( POC) testing helps the clinicians to start appropriate management for evolving state of affairs of the patients, thereby this would be beneficial both clinically and economically for the patient. Electrolyte values are measured for all critically ill patients in pediatrics emergency department and also for patients given fluid therapy or patients admitted in pediatric intensive care units. ${ }^{4}$ Serum electrolytes measurement in the main central Laboratory of the hospital is technically more difficult and extensive. A large number of serious patients are received in emergency centers of tertiary. Hospitals, serum electrolytes are measured by ABGs analyzers, because it is easier and quicker. ${ }^{5}$ A study found that the difference between ABGs and laboratory serum value of sodium was found to be $4 \pm 5.6 \mathrm{mmol} / 1$ and for potassium, $-1 \pm 0 \mathrm{mmol} / 1$

1.The Children's Hospital \& The Institute of Child Health, Lahore, Pakistan.

Correspondence: Dr. Aaqib Javed, Post Graduate Resident in pediatric Medicine CH\&ICH, Lahore. Pakistan.

Email: draqibm@gmail.com

Received: 29-01-2020 Published: 08-06-2021 
$(n=35)$. The difference between ABGs and laboratory serum levels for both electrolytes was significant $(\mathrm{p}<0.05)$ and ABGs showed fewer values as compared to laboratory serum levels. ${ }^{6}$ Another study found that the difference between ABGs and laboratory serum value of sodium was $4.1 \pm 0.3 \mathrm{mmol} / 1$ and for potassium, $-0.3 \pm 0.2$ $\mathrm{mmol} / \mathrm{l}(\mathrm{n}=44)$. The difference between ABGs and laboratory serum levels for both electrolytes was significant $(\mathrm{p}<0.05)$ and ABGs showed fewer values as compared to laboratory serum levels. ${ }^{7}$

Serum evaluation through laboratory investigations is costly and requires time much more than investigations through ABGs. Studies have shown that there is a difference in levels measured through both methods. But above studies were conducted on a small sample size. We conducted this study on a large sample size to get more reliable results. This will help to improve our practice and we will be able to implement the use of the best diagnostic method which will be more cost and time-effective. The objective of this study was to compare the difference in electrolyte level in arterial blood gases level versus laboratory serum electrolyte level in children admitted to the pediatric intensive care unit.

\section{Methodology}

This cross-sectional study was conducted at the department of Pediatrics, the Children's Hospital and Institute of Child Health, Lahore, over a period of 6 months from $1^{\text {st }}$ December 2015 to $31^{\text {st }}$ May 2016. A total sample of 125 children nonprobability, consecutive sampling with 95\% confidence level, $\mathrm{d}=1$ of test and taking the magnitude of mean difference of sodium level i.e. $4 \pm 5.6 \mathrm{mmol} / 1$ in ABGs level and fulfilling inclusion criteria, were enrolled in the study from pediatric intensive care unit. Informed consent was obtained from parents. Children of age range 1 year to 15 years of either gender admitted to Pediatric intensive unit for hypotension, diabetic ketoacidosis, kidney dysfunction. Patients were grouped in different ages i.e. 1 to 5 years, 6 to 10 years, and 11 to 15 years. Children with severe dehydration $(>7 \%$ of the difference between the current body weight and the immediate premorbid weight) and children with severe malnutrition (-2sd from normal value for specific age as per who criteria) were excluded.
Demographic profile was also noted. Children were admitted in PICU. After aseptic techniques, a venous blood sample (2cc) and arterial blood sample (1cc) was obtained from the radial or brachial artery (preferably radial), using a 24 gauge needle. The ABGs electrolytes were obtained immediately after sample collection, using ABGs analyzer. Serum electrolytes were analyzed in the main laboratory of the institution. Reports were assessed and levels of sodium and potassium were noted from reports of ABGs and laboratory. The difference was calculated. Data was entered and analyzed by SPSS V-20. Quantitative variables like age and electrolyte level by ABGs, serum electrolyte, and the difference was calculated as mean and standard deviation. Qualitative variables like gender were calculated as frequency and percentage. The difference between mean electrolyte levels (sodium and potassium levels) was calculated by subtracting laboratory values from ABGs. Data was stratified for age, gender to deal with effect modifiers. Poststratification, t-test was applied. P-value $\leq 0.05$ was taken as significant.

\section{Results}

The mean age of children included in this study was $6.72 \pm 4.02$ years. Range was from 1 to 15 years. Gender distribution of children showed that there were 63(50.40\%) males and 62(49.60\%) females. (male and female ratio). ${ }^{\text {The mean } \mathrm{Na}+}$ value from ABGs and from the laboratory was $134.66 \pm 8.577$ and 132.26 \pm 8.70 . The mean difference in both these values was $2.40 \pm 1.55$. However $\mathrm{NA}^{+}$values obtained from ABGs were higher than that of laboratory values $(p=0.016)$. The mean $\mathrm{K}^{+}$value from $\mathrm{ABG}$ and from laboratory was $4.51 \pm 0.90$ and $4.28 \pm 0.93$. The mean difference in both these values was $0.230 \pm 0.16$. However $\mathrm{K}^{+}$values obtained from ABGs were higher than that of laboratory values.

Table-I: Statistics for sodium $\mathrm{Na}^{+}$and $\mathrm{K}^{+}$by ABG and Laboratory methods

\begin{tabular}{|c|c|c|c|c|}
\hline Electrolytes & ABG's Value & $\begin{array}{c}\text { Laboratory } \\
\text { value }\end{array}$ & $\begin{array}{c}\text { Differe } \\
\text { nce }\end{array}$ & p-value \\
\hline $\mathbf{N a}^{+}$ & $134.66 \pm 8.57$ & $132.26 \pm 8.7$ & 2.40 & 0.016 \\
\hline $\mathbf{K}^{+}$ & $4.512 \pm 0.9$ & $4.282 \pm 0.93$ & 0.230 & 0.073 \\
\hline
\end{tabular}

Children in age group 1-5 years among them mean $\mathrm{NA}^{+}$value from ABGs was higher but not statistically significant ( $p$-value 0.064 ), while in age group 6-10 years and $11-15$ years it was significantly higher, i-e P-value (6-10 years) $=0.016 \&$ p-value 
$(11-15$ years $)=0.008$. Among male and female children the value of $\mathrm{NA}^{+}$was significantly higher from $\mathrm{ABG}$ as compared to that of values of $\mathrm{Na}+$ checked in lab, i.e. P-value (male) $=0.032 \&$ pvalue $($ female $)=0.026$. $($ Table-II $)$

Table-II: Descriptive Statistics for Sodium $\mathrm{Na}^{+}$ in relation to Age and Sex of Children

\begin{tabular}{|l|c|c|c|}
\hline Na+ Level & 1 -5 Years & 6-10 Years & 11 -15 Years \\
\hline ABG's Value & $135.21 \pm 8.76$ & $133.18 \pm 8.94$ & $135.93 \pm 7.51$ \\
\hline $\begin{array}{l}\text { Laboratory } \\
\text { value }\end{array}$ & $133.13 \pm 9.04$ & $130.45 \pm 8.96$ & $133.43 \pm 7.39$ \\
\hline Difference & $\mathbf{2 . 0 8} \pm 1.45$ & $\mathbf{2 . 7 3} \pm 1.60$ & $\mathbf{2 . 5 0} \pm 1.57$ \\
\hline p-value & $\mathbf{0 . 0 6 5 8}$ & $\mathbf{0 . 0 1 6}$ & $\mathbf{0 . 0 0 8}$ \\
\hline Na+ Level & Male & Female & \\
\hline ABG's Value & $134.76 \pm 9.05$ & $134.55 \pm 8.13$ & \\
\hline $\begin{array}{l}\text { Laboratory } \\
\text { value }\end{array}$ & $132.30 \pm 9.06$ & $132.21 \pm 8.40$ & \\
\hline Difference & $\mathbf{2 . 4 6} \pm 1.64$ & $\mathbf{2 . 3 4} \pm 1.45$ & \\
\hline p-value & $\mathbf{0 . 0 3 2}$ & $\mathbf{0 . 0 2 6}$ & \\
\hline
\end{tabular}

Children in age group 1-5 years and 6-10 years among them mean $\mathrm{K}^{+}$value from $A B G s$ was higher but not statistically significant $\mathrm{p}$-value (1-5 years $)=0.065, p$-value $(6-10$ years $)=0.073$. However, in the age group 11-15 years it was significantly higher, i.e. P-value (11-15 years) $=0.014$. Among male children, the value of $\mathrm{K}^{+}$was significantly higher from $\mathrm{ABG}$ as compared to that of values of $\mathrm{NA}^{+}$checked in the laboratory. However among female children value of $\mathrm{K}^{+}$was higher but not significant from $\mathrm{ABG}$ i.e. P-value $($ male $)=0.029 \&$ p-value $($ female $)=0.071$. (Table-III)

Table-III: Descriptive statistics for Potassium $\mathrm{K}^{+}$in relation to Age and Sex of Children

\begin{tabular}{|l|c|c|c|}
\hline K+ Level & $1-5$ Years & $6-10$ Years & $11-15$ Years \\
\hline ABG's Value & $4.45 \pm 0.92$ & $4.56 \pm 0.93$ & $4.54 \pm 0.84$ \\
\hline Laboratory value & $4.23 \pm 0.96$ & $4.35 \pm 0.92$ & $4.27 \pm 0.89$ \\
\hline Difference & $\mathbf{0 . 2 2} \pm 0.16$ & $\mathbf{0 . 2 1} \pm 0.15$ & $\mathbf{0 . 2 7} \pm 0.17$ \\
\hline p-value & $\mathbf{0 . 0 6 5}$ & $\mathbf{0 . 0 7 3}$ & $\mathbf{0 . 0 1 4}$ \\
\hline K+ Level & Male & Female & \\
\hline ABG's Value & $\mathbf{4 . 5 8} \pm 0.85$ & $\mathbf{4 . 4 3} \pm 0.95$ & \\
\hline Laboratory value & $\mathbf{4 . 3 4} \pm \mathbf{0 . 8 8}$ & $\mathbf{4 . 2 1} \pm 0.97$ & \\
\hline Difference & $\mathbf{0 . 2 3 5} \pm 0.16$ & $\mathbf{0 . 2 2} \pm 0.16$ & \\
\hline p-value & $\mathbf{0 . 0 2 9}$ & $\mathbf{0 . 0 7 1}$ & \\
\hline
\end{tabular}

\section{Discussion}

Skin, a homeostatic and protective organ in its Electrolyte abnormalities can be fatal. Quick and precise measurements of electrolyte derangements make the possibility of targeted therapies the institution. The quickness of such measurement, particularly in underdeveloped countries, is often delayed in the transport of samples to the main laboratory of the institution, either due to the inadequate numbers of human resources or the lack of quick transportation systems. ${ }^{7}$ There are usually results in longer time duration usually longer than one hour may be up to 2 hours to obtain serum electrolytes in main central laboratories of hospitals. These delays in serum measurements have major impact on patient's prognosis. The point-of-care tests have been used in the intensive care units also in the emergency department that make the possibility of rapid assessment of $\mathrm{ABG}$ and electrolyte abnormalities.

In this study mean $\mathrm{Na}^{+}$value on $\mathrm{ABG}$ and from the laboratory was 134.66 and 132.26. The mean difference in both these values was 2.40 . The mean $\mathrm{K}^{+}$value from on $\mathrm{ABG}$ and from the laboratory was 4.512 and 4.282. The mean difference in both these values was 0.230 . Both these electrolytes were seen in relation to the age and gender of children. Except age group 1-5 years statistically significant difference was seen for $\mathrm{Na}^{+}$in other two age groups i.e. 6-10 (p-value $=0.016)$ and 11-15 years $(p-$ value $=0.008)$ respectively.

The same trend was observed in male and female children that mean $\mathrm{Na}^{+}$and $\mathrm{K}^{+}$(except female) value with $\mathrm{ABGs}$ was significantly higher as compared to that of laboratory value. $\left(\mathrm{Na}^{+}\right.$level) male: [ABGs: 134.76 vs. Laboratory value: 132.30 , p-value $=0.032]$ female: [ABGs: 134.55 vs. Laboratory value: 132.21, p-value $=0.026$ ]. $\left(\mathrm{K}^{+}\right.$level $)$male: [ABGs: 4.58 vs. Laboratory value: $4.34, \mathrm{p}$-value $=0.029$ ] female: [ABGs : 4.43 vs. Laboratory value: 4.21, pvalue $=0.071]$.

Razavi, s. Et al, ${ }^{6}$ compared sodium and potassium concentrations between serum from venous blood and plasma from arterial blood. As per his findings mean serum concentrations of sodium ( $\mathrm{NA}^{+}$ $\mathrm{v}=137.1 \pm 5.5)$ and potassium $\left(\mathrm{K}^{+} \quad \mathrm{v}=4.1 \pm 0.7\right)$ were higher than plasma concentrations of sodium $\left(\mathrm{NA}^{+}\right.$ $\mathrm{a}=133.1 \pm 11.1) ; \mathrm{p}<0.02$ and potassium $\left(\mathrm{K}^{+} \quad \mathrm{v}\right.$ $=3.1 \pm 0.7) ; \mathrm{p}<0.001$ respectively. ${ }^{6}$ The same findings were seen in our study as that reported by Razavi, S, et al. Mean $\mathrm{Na}^{+}$and $\mathrm{K}^{+}$values from arterial blood gases on ABGs were higher than that of the laboratory values.

Maher Khader et al, ${ }^{10}$ determine whether the levels of 
blood electrolytes assessed using a blood gas analyzer and central laboratory auto-analyzer are equivalent. As per his findings, sodium and potassium levels measured by blood gas analyzer and auto-analyzer were significantly different. The mean level of sodium and potassium measured by the blood gas analyzer was significantly higher than in the auto-analyzer $\left(\mathrm{Na}^{+}: 141.4 \pm 7.3 \&\right.$ and $137.7 \pm 5.6$ respectively; $\mathrm{p}<0.001)\left(\mathrm{K}^{+} 3.2 \pm 0.8 \& 4.2 \pm 0.7 ; \mathrm{p}<0.001\right) .48$ his results also support the results of our study as the mean value of $\mathrm{Na}^{+}$and $\mathrm{K}^{+}$were higher in arterial blood gases assessed on ABGs as compared to that o laboratory values. ${ }^{9,10}$ As per findings of Alnazi, A, et al, critical decisions can be made according to values obtained through both ABGs and serum levels of the electrolytes as there is no gross difference between them."

Nanda, SK et al, concluded that sodium and potassium analyzed in point of care ABGs analyzer is comparable to the sodium and potassium levels measured in electrolyte analyzer and ABGs electrolytes can be used as a replacement to electrolytes measured in electrolyte analyzer. (mean arterial sodium: 134 \& mean venous sodium 137, mean arterial potassium: $3.6 \&$ mean venous potassium: 4.1$).{ }^{12}$ In a study by Chacko, $\mathrm{B},{ }^{7}$ et al, there is a clinically major differentiation between electrolytes measured by serum samples at the main laboratory of the hospital and value obtained from blood samples measured at the point of care.

Our study is comparable with other studies which found significantly different results for serum sodium and potassium values obtained using two different types of measurements. ${ }^{13-15}$ some of the authors determined a correction factor to reduce the different results between the two analyzer machines by making compensation at an average level. ${ }^{16-18}$ On contrary to the current study, Jain A, et al, ${ }^{4}$ found there is no gross difference between electrolytes measured by ABGs analyzers and serum auto analyzers. They concluded in their study that prompt decisions can be made according to the potassium level obtain from the ABGs analysis. ${ }^{14}$

The significant differences and poor correlation between sodium and potassium values measured by blood glucose analyzer and auto analyzer may be attributed to several factors, including the type of sample used (whole blood vs. Serum), preanalytic dilution of serum samples with fixed volume diluents (indirect vs. Direct electrodes), and variations in calibration of the machine. ${ }^{18-20}$ Another factor that contributes is the dilution of the sample by using heparin that is used in syringes. Experts have theoretically considered that heparin diluted samples have a lower level of electrolytes. ${ }^{21,22}$ All these factors may vary from place to place i.e. instrument type, calibration of the machine. It is essential that each center conduct its own study and derives a correct factor that may be applied if different methods of assessment are used. The difference in serum and whole blood electrolytes results is because of the different contributory factors such as type of samples (whole blood vs. Serum), variation in the calibration of the machine as well as serum sample dilution. By using a correction factor Variations as a result of these factors can be compensated.

Application of a correction factor is commonly used now a day. According to the international federation of clinical chemistry (IFCC) constant conversion factor (of 1.11) between blood and plasma glucose to provide "harmonized results", that facilitate the classification and patients care and leading to lesser therapeutic errors, however, the correction factor for electrolytes may need to be generated for each center.

\section{Conclusion}

The serum electrolytes $\mathrm{Na}^{+}$and $\mathrm{K}^{+}$values from arterial blood gases level were different from laboratory serum electrolyte level in children admitted to a pediatric intensive care unit. Therefore the data can't be used in clinical practice. The clinician should be aware of differences so that potential misdiagnosis does not occur and unnecessary treatment or investigation can't be performed.

Authors Contribution: HMSS: Conception of work and Drafting. MUS: Acquisition and Analysis of data and Revising. SF: Design of work and Revising. SA: Interpretation of data and Drafting. AJ: Analysis of data and drafting. AMA: Design of work and Drafting. All authors critically revised and approve its final version.

Conflict of Interest: Authors has declared no conflict of interest.

Sources of Funding: The source of funding was self. Disclaimer: None 


\section{References}

1. Fiallos MR, Hanhan UA, Orlowski JP. Point-of-care testing. Pediatr Clin 2001;48(3):589-600.

2. Labovitz AJ, Noble VE, Bierig M, Goldstein SA, Jones $\mathrm{R}$, Kort S, et al. Focused cardiac ultrasound in the emergent setting: a consensus statement of the American Society of Echocardiography and American College of Emergency Physicians. J Am Soc Echocardiogr 2010;23(12):1225-30.

3. Mayo PH, Beaulieu Y, Doelken P, Feller-Kopman D, Harrod C, Kaplan A, et al. American College of Chest Physicians/La Société de Réanimation de Langue Française statement on competence in critical care ultrasonography. Chest J 2009;135(4):1050-60.

4. Jain A, Subhan I, Joshi M. Comparison of the point-ofcare blood gas analyzer versus the laboratory autoanalyzer for the measurement of electrolytes. Int J Emerg Med 2009;2(2):117-20.

5. Robles FC, Laguna Neto D, Dias FG, Spressão M, Matos PN, Cordeiro JA, et al. Diabetic ketoacidosis: the difference between potassium determined by blood gas analysis versus plasma measurement. Arq Bras Endocrinol Metabol 2011;55(4):256-9.

6. Razavi S, Jafari A, Zaker H, Sadeghi A. Plasma and Serum Electrolyte Levels Correlation in the Pediatric ICU. Tanaffos 2010;9(4):34-8.

7. Chacko B, Peter JV, Patole S, Fleming JJ, Selvakumar R. Electrolytes assessed by point-of-care testing-Are the values comparable with results obtained from the central laboratory? Indian J Crit Care Med 2011;15(1):24.

8. Cox CJ. Acute care testing. Blood gases and electrolytes at the point of care. Clinics in laboratory medicine 2001;21(2):321-35.

9. Mirzazadeh M, Morovat A, James T, Smith I, Kirby J, Shine B. Point-of-care testing of electrolytes and calcium using blood gas analysers: it is time we trusted the results. Emergency Medicine Journal 2015:emermed-2015-204669.

10. Maher khader AA-I, Fatin Al -Awaisha, Sameera Abuzeat RN, Asma Al Nesraween RN, Tagreed ALkasassbah RN. Are the electrolytes values of blood gas analyzer and a laboratory auto-analyzer comparable? Jour of Med Sc \& Tech 2014;3(2):57-60.

11.Alanazi A, Al Obaidi NY, Enezi FA, AlMutairi MK, Aljerian N, Alsultan OA, et al. Correlation between the Measurements of Serum and Arterial Blood Gas (ABG) Electrolytes in Patients Admitted to the Intensive Care Unit at King Abdul-Aziz Medical City, Riyadh, Saudi Arabia. American Journal of Clinical Medicine Research 2015;3(3):55-9
12. Nanda SK, Ray L, Dinakaran A. Agreement of arterial sodium and arterial potassium levels with venous sodium and venous potassium in patients admitted to the intensive care unit. Journal of clinical and diagnostic research: JCDR 2015;9(2):BC28.

13. Shalini Gupta, Ashwani K. Gupta, Kamaljit Singh, Minni Verma. Are sodium and potassium results on arterial blood gas analyzer equivalent to those on electrolyte analyzer? Indian J Crit Care Med. 2016 Apr; 20(4): 233-237.DOI:10.4103/0972-5229.180044

14. Jain A, Subhan I, Joshi M. Comparison of the point-of-care blood gas analyzer versus the laboratory auto-analyzer for the measurement of electrolytes. International $\mathrm{J}$ of Emerg Med 2009;2(2):117-20

15. Hrabrić Vlah S, Dvornik Š, Grdović D. Analytical performance of the Gem ${ }^{\circledR}$ Premier ${ }^{\mathrm{TM}}$ 4000-a comparison study. Biochemia Medica 2009;19(2):192-8.

16. Chow E, Fox N, Gama R. Effect of low serum total protein on sodium and potassium measurement by ion-selective electrodes in critically ill patients. British journal of biomedical science 2008;65(3):128.

17.D'Orazio P, Burnett RW, Fogh-Andersen N, Jacobs E, Kuwa $\mathrm{K}$, Külpmann WR, et al. Approved IFCC recommendation on reporting results for blood glucose (abbreviated). Clinical chemistry 2005;51(9):1573-6.

18. Budak YU, Huysal K, Polat M. Use of a blood gas analyzer and a laboratory autoanalyzer in routine practice to measure electrolytes in intensive care unit patients. BMC anesthesiology 2012;12(1): 1'

19.Prichard JS, French JS, Alvar N. Clinical evaluation of the ABL-77 for point-of-care analysis in the cardiovascular operating room. The Journal of extra-corporeal technology 2006;38(2): 128 .

20.Chacko B, Peter JV, Patole S, Fleming JJ, Selvakumar R. Electrolytes assessed by point-of-care testing-Are the values comparable with results obtained from the central laboratory Indian Journal of Critical Care Medicine 2011;15(1):24.

21. Van Berkel M, Scharnhorst V. Electrolyte-balanced heparin in blood gas syringes can introduce a significant bias in the measurement of positively charged electrolytes. Clinical Chemistry and Laboratory Medicine 2011;49(2):249-52.

22.Lima-Oliveira G, Lippi G, Salvagno GL, Montagnana M, Picheth G, Guidi GC. Different manufacturers of syringes: A new source of variability in blood gas, acid-base balance and related laboratory test? Clinical biochemistr2012; 45(9):683-7 